\title{
CEP APPLICATION IN AN ENERGY MANAGEMENT EQUIPMENT MAINTENANCE INDUSTRY REMOTELY
}

\author{
Claudionor Monteiro Ramos ${ }^{1}$, Danyanne da Silva Corrêa ${ }^{2}$, \\ Eliana da Conceição Rodrigues Veras ${ }^{3}$, Simone Silva de Arruda Souza ${ }^{4}$
}

\begin{abstract}
${ }^{1}$ Ingeniero Eléctrico, alumno del Curso de Maestría en Ingeniería de Procesos de la UFPA.
${ }^{2}$ Administradora, Especialista en Auditoría Contable, Fiscal y Tributaria alumna del Máster en Ingeniería de Procesos de la UFPA.

${ }^{3}$ Administradora, Especialista en Metodología de la Enseñanza Superior, Maestría en Gestión y Auditoría Ambiental, alumna del Máster en Ingeniería de Procesos de la UFPA.

${ }^{4}$ Administradora, Especialista en Gestión de Personas, alumna del Curso de Maestría en Ingeniería de Procesos de la UFPA.
\end{abstract}

Email: claudionor.ramos@hotmail.com, danyanne.correa@gmail.com, elianaveras8@gmail.com simonearrudacoach@gmail.com

Received: April 29th, 2019

Accepted: May $27^{\text {th }}, 2019$

Published: September $30^{\text {th }}$, 2019

Copyright @ 2016 by authors and Institute of Technology Galileo of Amazon (ITEGAM). This work is licensed under the Creative Commons Attribution International License (CC BY 4.0). https://creativecommons.org/lice nses/by/4.0/

\begin{abstract}
The research is applied to the Statistical Control of Process-SPC in a micro-enterprise that performs maintenance services on remote energy management equipment, installed in the Industrial Pole of Manaus-PIM. There was an increase in the return of the equipment sent within the maintenance period, where adjustments of improvements were necessary. The goal will be to apply control charts Control $\mathrm{P}$ and NP and Pareto chart. Based on the data graphs, the control chart P was applied because it was necessary to analyze the defective items. In the evaluation of the capacity of the process, the letter NP was used, because it was aimed to know the number of defective items. The causes of nonconformities found in the process were solved as they were identified, in a way that met the needs of the client and the objectives of the company. The quality adjustments with the implantation of the CEP in the company, make that the products today are meeting the specifications.
\end{abstract}

Keywords: SPC; Conformity; Control.

\section{APLICACIÓN DEL CEP EN UNA INDUSTRIA DE MANTENIMIENTO DE EQUIPOS DE GESTIÓN DE ENERGÍA DE FORMA REMOTA}

\section{RESUMEN}

La investigación se destina a la aplicación del Control Estadístico del Proceso-CEP en una microempresa que realiza servicios de mantenimiento en equipos de gestión de energía de forma remota, instalada en el Polo Industrial de Manaus-PIM. Se observó un aumento en el retorno de los equipos enviados dentro del plazo de la manutención, donde fueron necesarias adecuaciones de mejoras. El objetivo será aplicar las cartas de control Control P y NP y gráfico de Pareto. Basándose en los gráficos de datos, se aplicó la carta de control $\mathrm{P}$, pues fueron necesarios analizar los ítems con defectos. En la evaluación de la capacidad del proceso, se utilizó la carta NP, pues se objetivó conocer el número de ítems defectuosos. Las causas de no conformidades encontradas en el proceso, fueron solucionadas a medida que fueron identificadas, de cierta forma se atendieron las necesidades del cliente y los objetivos de la empresa. Los ajustes de calidad con la implantación del CEP en la empresa, hacen que los productos hoy estén atendiendo a las especificaciones.

Palavras chave: Palabras clave: CEP; cumplimiento; Control. 


\section{INTRODUCCIÓN}

Las empresas de prestación de servicios vienen buscando aumentar la calidad de sus procesos en los últimos años, ya que es un factor determinante en su éxito o fracaso. Es de notar que garantizar una buena calidad en el servicio no es una tarea fácil, pues la mayoría de las veces, aun ejecutando acciones en sus procesos internos, no se tiene la garantía de que esto reflejará en la satisfacción del cliente. No hay garantía de que los servicios siempre se presten en el mismo nivel planificado. Los servicios sufren muchas influencias que afectan al estándar de calidad: las personas, la cultura de la empresa en relación a los clientes y el trato de los empleados, el liderazgo practicado por cada gestor de la línea de frente y los diversos perfiles de clientes con sus opiniones y necesidades diferente.

Los métodos estadísticos se pueden utilizar en las distintas fases del proceso del servicio para minimizar y mantener bajo control la calidad de los servicios.

El Control Estadístico del Proceso (CEP) es una colección de herramientas, que auxiliando en la disminución de la variabilidad del proceso, permiten el alcance de un proceso estable cuya capacidad puede ser mejorada.

En el artículo se presenta la aplicación del CEP, en la empresa "X", que posee 13 tiendas de mantenimiento de equipos de empresas instaladas en el Polo Industrial de Manaus - PIM. En el caso de que se produzca un cambio en el rendimiento de los equipos dentro del plazo de garantía de mantenimiento, se requiere una adecuación para el servicio al cliente.

A partir de la aplicación del CEP, se detectaron las fallas y sus causas, proporcionándonos informaciones que ayudaron en la toma de decisión.

El objetivo del artículo es presentar el resultado del estudio de caso sobre la aplicación del CEP en la microempresa X, utilizando las cartas de control desarrolladas a partir de la necesidad del estudio de datos del proceso resultante del mantenimiento, donde no había controles.

La aplicación del CEP en microempresas es algo desafiante en la realización de la investigación, que puede ser referencia en el futuro. Se eligieron muestras $\mathrm{N}$ de un universo X, es decir, 200 muestras cada 2000 equipos entregados. Se utilizaron cartas de control tipo P y NP, Diagrama de flujo y Gráfico de Pareto para el análisis de los problemas.

Se espera de esta manera ser un referencial para implantación del CEP en industrias afines desta maneira ser um referencial para implantação do CEP em indústrias afins.

\section{FUNDAMENTACIÓN TEÓRICA}

El Control Estadístico del Proceso contiene algunos pasos para la organización y control del proceso, que según [1], los pasos para la ejecución del CEP, que son: la recolección y el trazado de los datos; las pruebas de control; la investigación de las causas atribuibles; la aplicación de mejoras; y la eliminación de los datos influenciados por causas atribuibles. Todas las empresas pueden aplicarlo en sus procesos de forma que el CEP puede sí mejorar el proceso dentro de su especificidad.

El CEP ha sido utilizado en muchas industrias como forma de aplicación para controlar sus procesos y mejorar sus actividades, con los resultados las decisiones de mejora se vuelven más precisas, según [2], los principales objetivos del CEP son una identificación de la variabilidad anormal causada por causas con el objetivo de: 1. hacer el proceso estable; 2 . minimizar la variabilidad del proceso y 3. mejorar el rendimiento del proceso. El CEP no debe ser aplicado solamente, pero se debe haber una continuidad de ese control, para no ser necesario realizar implementaciones después de resultados sin control, o por dejar de aplicarlos, tornarse rutinario para siempre haber prevención de los posibles problemas.

El levantamiento de los datos nos llevó a determinar que los gráficos serían por números de defectos y definir el número de muestras recolectadas. Según [3] En el caso de servicios, los datos importantes son los números de reclamaciones o devoluciones, por unidad de servicio. Donde se optó por la Carta P y la Carta NP.

Para que pudiéramos atender las especificaciones del cliente, se estableció un parámetro, o más precisamente la tolerancia de un límite superior e inferior. Para [4] Las tolerancias establecen los límites superiores e inferiores de especificación, y se definen en el departamento de proyecto oa través de los requisitos de los clientes.

Cuando los productos no se ajustan a las especificaciones, para su mejor visualización se deben aplicar gráficos que indican sus errores donde pueden ser definidos los límites para tomas de decisiones, según [5] o gráficos de control se utilizan para supervisar el rendimiento de un proceso de medición. Estos gráficos determinan estadísticamente una banda denominada límites de control, que está limitada por una línea superior (límite superior de control-LSC) y una línea inferior (límite inferior de control-LIC), además de una línea central (límite central LC).

La carta $\mathrm{P}$ se utiliza para demostrar productos o servicios como defectuosos o no defectuosos a través de gráficos donde se define el límite inferior y el superior de donde se aplicarán las correcciones para análisis, y de acuerdo con [6], los métodos estadísticos utilizados en ambas fases fueron las cartas de control por la media. Las convenciones utilizadas en la carta de control se describen como LIC y LSC, límites inferior y superior de control.

Para desarrollar planificaciones que maximicen la calidad y minimicen los problemas se debe aplicar un análisis de calidad para implementar proyectos de mejoras, para [7]:

"Al menos cinco razones para el uso de los gráficos de control, tales como: para mejorar la productividad; para la prevención de defectos; para impedir ajustes innecesarios en el proceso, ya que distingue entre el ruido aleatorio y la variación anormal; para proporcionar información para que el operador haga un diagnóstico sobre el proceso, pudiendo conducir la implantación de un cambio que mejore el desempeño del proceso; y proporcionar información sobre la capacidad del proceso, pues trae informaciones sobre el valor de importantes parámetros del proceso y su estabilidad a lo largo del tiempo.

En el análisis de los procesos y aplicación de las mejoras, los gráficos indican en la línea media los que no están fuera del control, pero no impide que éstos puedan ser monitoreados para su disminución, con [8] podemos afirmar que: Los gráficos de control auxilian la producción determinando si un proceso es estable o si existen causas especiales en la variación del proceso.

Los gráficos se dividen en Controles y Tributos, que permiten con el análisis de sus resultados la reducción de problemas, pues se aplican tomas de decisiones para resolución de defectos, y según Días [9], Gráficos de: El control se divide en dos categorías: Gráficos de Control para variables, aquel cuyo valor es determinado a través de algún tipo de medición y Gráficos de Control para atributos, determinado a través de una clasificación o conteo.

Al ser implantado el control podrá mantenerse un patrón controlado a fin de prevenir defectos, disminuir o estabilizarlos, aunque existen variabilidades que pueden ser muy grandes o estar fuera del estándar, afirma [10], que la implantación del CEP hará que el proceso permanezca estable y reducirá la variabilidad y de 
cierta forma en cualquier proceso por más cauteloso que sea variabilidad siempre existirá.

\section{METODOLOGÍA}

Al principio se elaboró el diagrama de flujo en el proceso, a fin de evaluar posibles fallas y que podrían resolverse antes de la aplicación de las cartas de control. De las 2000 unidades entregadas, 200 muestras fueron retiradas aleatoriamente para el proceso de evaluación, teniendo en cuenta que el cliente considera sólo el 10\% del cuantitativo aceptable, dentro de un período de 100 semanas, dentro del período de garantía.

La Tabla 1 muestra la información cuantitativa de la participación en la reparación por tienda durante las 100 semanas. Se nota que las tiendas $1,3,5,7,8,9,10$ y 13 tienen mayor cantidad de equipos por semana.

Tabla 1 - Levantamiento cuantitativo de unidad reparada semanal por tienda.

\begin{tabular}{|c|c|c|c|c|}
\hline \multicolumn{5}{|c|}{ 100 Semanas - Datos recopilados } \\
\hline Tiendas & S1 & S2 & .. & S100 \\
\hline 1 & 151 & 165 &.. & 142 \\
\hline 2 & 39 & 34 & $\cdots$ & 46 \\
\hline 3 & 246 & 210 & $\cdots$ & 227 \\
\hline 4 & 53 & 60 & $\cdots$ & 68 \\
\hline 5 & 234 & 272 & $\cdots$ & 214 \\
\hline 6 & 96 & 91 & $\cdots$ & 105 \\
\hline 7 & 308 & 323 & $\cdots$ & 317 \\
\hline 8 & 179 & 162 & $\cdots$ & 181 \\
\hline 9 & 247 & 227 & $\cdots$ & 220 \\
\hline 10 & 183 & 175 & $\cdots$ & 191 \\
\hline 11 & 76 & 56 & $\cdots$ & 45 \\
\hline 12 & 65 & 69 & $\cdots$ & 75 \\
\hline 13 & 176 & 141 & $\cdots$ & 168 \\
\hline Total & $\mathbf{2 0 5 3}$ & $\mathbf{1 9 8 5}$ & $\cdots$ & $\mathbf{1 9 9 9}$ \\
\hline
\end{tabular}

Fuente: Los Autores, (2019).

De los equipos elegidos para análisis, se verificó el cuantitativo de retornos con no conformidades, devueltos a la empresa dentro del plazo de garantía, más precisamente 1 año.
Tabla 2 - Cuantitativo de defectos de los equipos en plazo de garantía

\begin{tabular}{|c|c|c|c|c|c|}
\hline $\begin{array}{c}\text { Seman } \\
\text { a }\end{array}$ & $\begin{array}{l}\text { Defecto } \\
\text { s }\end{array}$ & $\begin{array}{l}\text { Grupo de } \\
\text { Muestras }\end{array}$ & Semana & $\begin{array}{c}\text { Defecto } \\
\text { s }\end{array}$ & $\begin{array}{l}\text { Grupo de } \\
\text { Muestras }\end{array}$ \\
\hline 1 & 6 & 200 & 51 & 2 & 200 \\
\hline 2 & 11 & 200 & 52 & 5 & 200 \\
\hline 3 & 3 & 200 & 53 & 3 & 200 \\
\hline 4 & 12 & 200 & 54 & 16 & 200 \\
\hline 5 & 6 & 200 & 55 & 8 & 200 \\
\hline 6 & 5 & 200 & 56 & 6 & 200 \\
\hline 7 & 1 & 200 & 57 & 1 & 200 \\
\hline 8 & 4 & 200 & 58 & 10 & 200 \\
\hline 9 & 4 & 200 & 59 & 2 & 200 \\
\hline 10 & 7 & 200 & 60 & 7 & 200 \\
\hline 11 & 15 & 200 & 61 & 6 & 200 \\
\hline 12 & 8 & 200 & 62 & 7 & 200 \\
\hline 13 & 6 & 200 & 63 & 1 & 200 \\
\hline 14 & 3 & 200 & 64 & 3 & 200 \\
\hline 15 & 5 & 200 & 65 & 9 & 200 \\
\hline 16 & 8 & 200 & 66 & 6 & 200 \\
\hline 17 & 9 & 200 & 67 & 4 & 200 \\
\hline 18 & 2 & 200 & 68 & 1 & 200 \\
\hline 19 & 4 & 200 & 69 & 3 & 200 \\
\hline 20 & 11 & 200 & 70 & 6 & 200 \\
\hline 21 & 4 & 200 & 71 & 3 & 200 \\
\hline 22 & 3 & 200 & 72 & 6 & 200 \\
\hline 23 & 3 & 200 & 73 & 10 & 200 \\
\hline 24 & 7 & 200 & 74 & 2 & 200 \\
\hline 25 & 1 & 200 & 75 & 6 & 200 \\
\hline 26 & 9 & 200 & 76 & 4 & 200 \\
\hline 27 & 6 & 200 & 77 & 9 & 200 \\
\hline 28 & 6 & 200 & 78 & 2 & 200 \\
\hline 29 & 6 & 200 & 79 & 1 & 200 \\
\hline 30 & 1 & 200 & 80 & 11 & 200 \\
\hline 31 & 11 & 200 & 81 & 1 & 200 \\
\hline 32 & 18 & 200 & 82 & 10 & 200 \\
\hline 33 & 5 & 200 & 83 & 2 & 200 \\
\hline 34 & 8 & 200 & 84 & 6 & 200 \\
\hline 35 & 7 & 200 & 85 & 9 & 200 \\
\hline 36 & 5 & 200 & 86 & 8 & 200 \\
\hline 37 & 8 & 200 & 87 & 1 & 200 \\
\hline 38 & 7 & 200 & 88 & 10 & 200 \\
\hline 39 & 1 & 200 & 89 & 4 & 200 \\
\hline 40 & 6 & 200 & 90 & 2 & 200 \\
\hline 41 & 12 & 200 & 91 & 2 & 200 \\
\hline 42 & 3 & 200 & 92 & 2 & 200 \\
\hline 43 & 9 & 200 & 93 & 4 & 200 \\
\hline 44 & 4 & 200 & 94 & 12 & 200 \\
\hline 45 & 4 & 200 & 95 & 2 & 200 \\
\hline 46 & 1 & 200 & 96 & 11 & 200 \\
\hline 47 & 2 & 200 & 97 & 4 & 200 \\
\hline 48 & 6 & 200 & 98 & 9 & 200 \\
\hline 49 & 8 & 200 & 99 & 11 & 200 \\
\hline 50 & 7 & 200 & 100 & 4 & 200 \\
\hline
\end{tabular}

Fuente: Los Autores, (2019).

Después del levantamiento de los datos, la identificación de la necesidad de evaluar el proceso y la utilización de las cartas de control, se verificó si el mismo atendía las especificaciones del cliente. 


\section{RESULTADOS}

Con base en las tablas de datos, se aplicó la carta de control $\mathrm{P}$, teniendo en vista que había la necesidad de evaluar la proporción de ítems defectuosos. Se utilizó la de atributos y no variables. La Figura 1 ilustra los resultados.

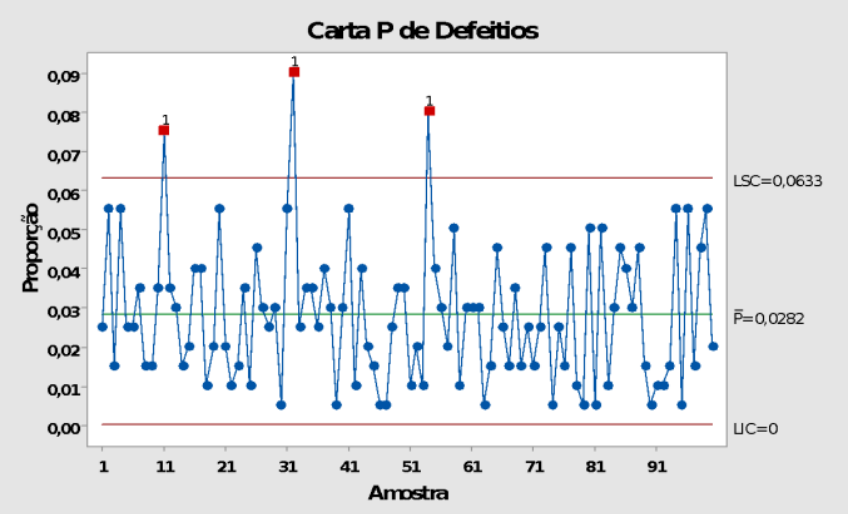

Figura 1 - Gráfico elaborado por los autores de la carta de control $\mathrm{P}$ aplicado a los defectos por grupos de muestras recolectados. Fuente: Los Autores, (2019).

En la figura 2 se muestran las muestras que quedaron fuera del rango de control, y que se eliminará: 11, 32 y 54
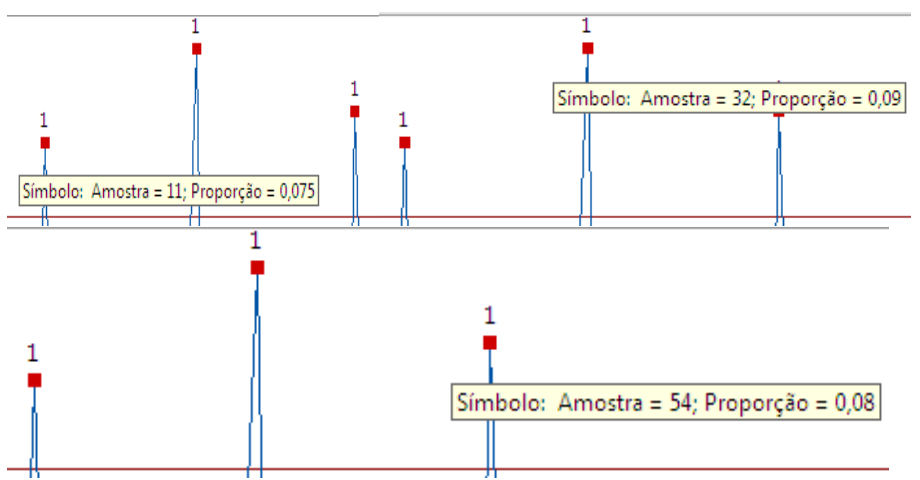

Figura 2 - Elaborado por los autores. Muestras eliminadas - 11, 32 y 54.

Fonte: Los Autores, (2019).

El gráfico de la carta de control con los datos restantes se muestra en la Figura 3. Se verifica en este caso que a partir de ahí el proceso queda bajo control estadístico.

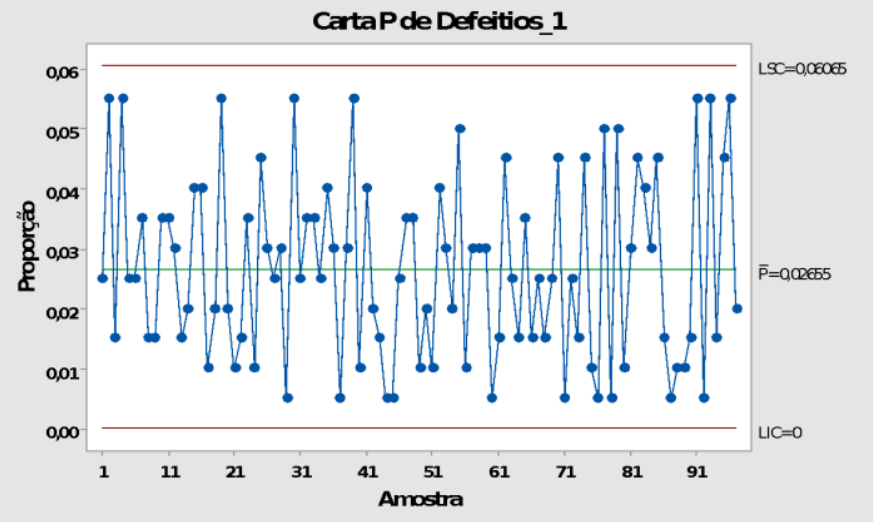

Figura 3 - Gráfico elaborado por los autores de la carta P aplicado a los datos restantes.
Fuente: Los Autores, (2019).

Para evaluar la capacidad del proceso, se debe satisfacer la especificación establecida por el cliente, que en este caso fue de hasta $10 \%$ de defectos para muestras de 200 recolectadas. El ideal en este caso es la utilización de la carta NP, pues se desea saber el número de ítems no conformes en lugar de la proporción de ítems defectuosos.

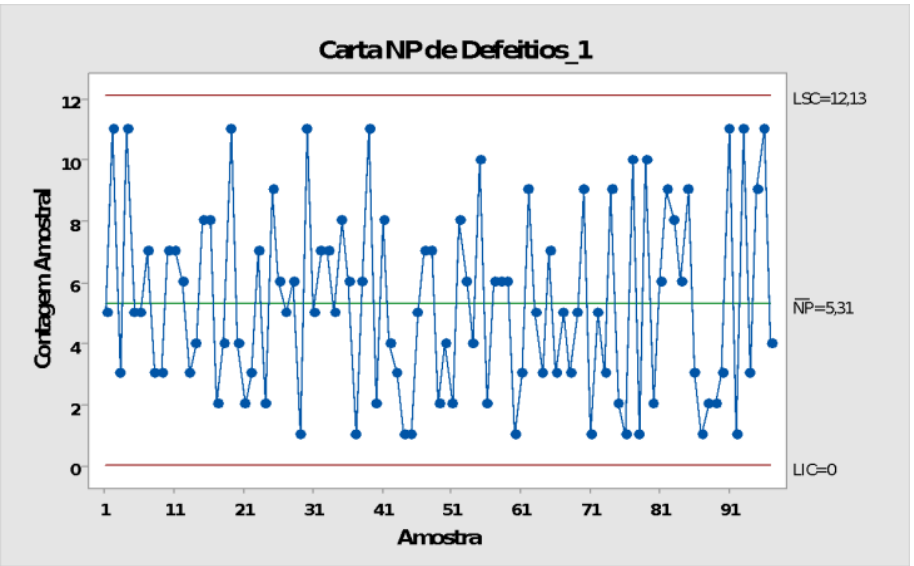

Figura 4 - Gráfico elaborado por los autores NP aplicado a los datos restantes.

Fuente: Los Autores, (2019).

Después de la retirada de los elementos defectuosos, se tiene el gráfico de la figura 4.

Si se establece el límite superior según la especificación, se obtiene el gráfico como se muestra en la Figura 5.

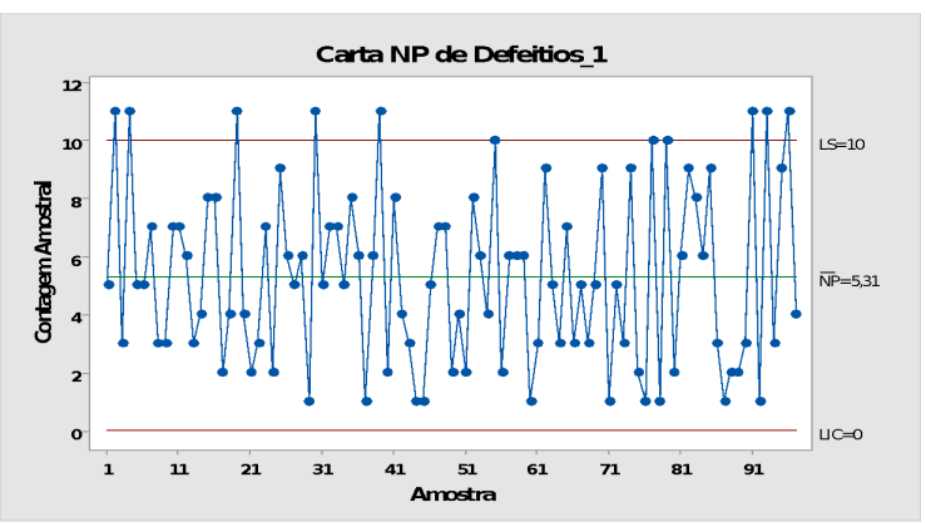

Figura 5 - Gráfico elaborado por los autores de la carta PN con límite superior igual a la especificación. Fuente: Los Autores, (2019).

Por la carta de control, verificamos que el sistema está bajo control estadístico, pero no es capaz, ya que las muestras 2, 4, 19, 30, 39, 91, 93 y 96 están fuera del límite establecido. En este caso, evaluados los posibles problemas, hizo observaciones de cambios en el proceso de mantenimiento para que atendieran las especificaciones del cliente.

En base a las muestras de defectos recogidos, se realizó la siguiente clasificación de defectos de los equipos.

Tipo 1 - SIMCARD (chip) no habilitado por el operador

Tipo 2 - SIMCARD (chip) defectuoso

Tipo 3 - Módulo de comunicación defectuoso

Tipo 4 - Instalación realizada de forma incorrecta

Tipo 5 - Antena desconectada 
Ramos et al., ITEGAM-JETIA. Vol. 05, No 19, pp 29-35. September, 2019.

Tabla 3 - Levantamiento cuantitativo de defecto por semana analizada.

\begin{tabular}{|c|c|c|c|c|c|c|c|}
\hline \multicolumn{8}{|c|}{ Tipo de defectos encontrados } \\
\hline Semana & Defeitos & $\begin{array}{c}\text { Tipo } \\
1\end{array}$ & $\begin{array}{c}\text { Tipo } \\
\mathbf{2}\end{array}$ & $\begin{array}{c}\text { Tipo } \\
\mathbf{3}\end{array}$ & $\begin{array}{c}\text { Tipo } \\
4\end{array}$ & $\begin{array}{c}\text { Tipo } \\
5\end{array}$ & Outros \\
\hline 1 & 5 & 2 & 1 & 1 & 0 & 1 & 0 \\
\hline 2 & 11 & 2 & 4 & 1 & 1 & 2 & 1 \\
\hline 3 & 3 & 0 & 1 & 0 & 1 & 1 & 0 \\
\hline 4 & 11 & 1 & 3 & 1 & 2 & 3 & 1 \\
\hline 5 & 5 & 0 & 2 & 1 & 1 & 1 & 0 \\
\hline 6 & 5 & 1 & 2 & 0 & 1 & 1 & 0 \\
\hline 7 & 7 & 1 & 3 & 2 & 0 & 1 & 0 \\
\hline 8 & 3 & 0 & 1 & 0 & 1 & 1 & 0 \\
\hline 9 & 3 & 0 & 1 & 0 & 1 & 1 & 0 \\
\hline 10 & 7 & 1 & 3 & 2 & 0 & 1 & 0 \\
\hline 11 & 15 & 3 & 5 & 3 & 1 & 2 & 1 \\
\hline 12 & 7 & 1 & 3 & 2 & 0 & 1 & 0 \\
\hline 13 & 6 & 1 & 2 & 1 & 1 & 1 & 0 \\
\hline 14 & 3 & 0 & 1 & 0 & 1 & 1 & 0 \\
\hline 15 & 4 & 1 & 1 & 1 & 0 & 1 & 0 \\
\hline 16 & 8 & 2 & 3 & 1 & 0 & 2 & 0 \\
\hline 17 & 8 & 2 & 3 & 1 & 0 & 2 & 0 \\
\hline 18 & 2 & 0 & 1 & 1 & 0 & 0 & 0 \\
\hline 19 & 4 & 1 & 1 & 1 & 0 & 1 & 0 \\
\hline 20 & 11 & 2 & 4 & 1 & 1 & 2 & 1 \\
\hline 21 & 4 & 1 & 1 & 1 & 0 & 1 & 0 \\
\hline 22 & 2 & 0 & 1 & 1 & 0 & 0 & 0 \\
\hline 23 & 3 & 0 & 1 & 0 & 1 & 1 & 0 \\
\hline 24 & 7 & 1 & 3 & 2 & 0 & 1 & 0 \\
\hline 25 & 2 & 0 & 1 & 1 & 0 & 0 & 0 \\
\hline 26 & 9 & 2 & 3 & 1 & 1 & 2 & 0 \\
\hline 27 & 6 & 1 & 2 & 1 & 1 & 1 & 0 \\
\hline 28 & 5 & 2 & 1 & 1 & 0 & 1 & 0 \\
\hline 29 & 6 & 1 & 2 & 1 & 1 & 1 & 0 \\
\hline 30 & 1 & 0 & 0 & 0 & 0 & 1 & 0 \\
\hline 31 & 11 & 2 & 4 & 1 & 1 & 2 & 1 \\
\hline 32 & 18 & 2 & 6 & 2 & 3 & 4 & 1 \\
\hline 33 & 5 & 2 & 1 & 1 & 0 & 1 & 0 \\
\hline 34 & 7 & 1 & 3 & 2 & 0 & 1 & 0 \\
\hline 35 & 7 & 1 & 3 & 2 & 0 & 1 & 0 \\
\hline 36 & 5 & 2 & 1 & 1 & 0 & 1 & 0 \\
\hline 37 & 8 & 2 & 3 & 1 & 0 & 2 & 0 \\
\hline 38 & 6 & 1 & 2 & 1 & 1 & 1 & 0 \\
\hline 39 & 1 & 0 & 1 & 0 & 0 & 0 & 0 \\
\hline 40 & 6 & 1 & 2 & 1 & 1 & 1 & 0 \\
\hline 41 & 11 & 2 & 4 & 1 & 1 & 2 & 1 \\
\hline 42 & 2 & 0 & 1 & 1 & 0 & 0 & 0 \\
\hline 43 & 8 & 2 & 3 & 1 & 0 & 2 & 0 \\
\hline 44 & 4 & 1 & 1 & 1 & 0 & 1 & 0 \\
\hline 45 & 3 & 0 & 1 & 0 & 1 & 1 & 0 \\
\hline 46 & 1 & 0 & 0 & 0 & 1 & 0 & 0 \\
\hline 47 & 1 & 0 & 1 & 0 & 0 & 0 & 0 \\
\hline 48 & 5 & 2 & 1 & 1 & 0 & 1 & 0 \\
\hline 49 & 7 & 1 & 3 & 2 & 0 & 1 & 0 \\
\hline 50 & 7 & 1 & 3 & 2 & 0 & 1 & 0 \\
\hline 51 & 2 & 0 & 1 & 1 & 0 & 0 & 0 \\
\hline 52 & 4 & 1 & 1 & 1 & 0 & 1 & 0 \\
\hline 53 & 2 & 0 & 1 & 1 & 0 & 0 & 0 \\
\hline 54 & 16 & 3 & 4 & 2 & 2 & 4 & 1 \\
\hline 55 & 8 & 2 & 3 & 1 & 0 & 2 & 0 \\
\hline 56 & 6 & 1 & 2 & 1 & 1 & 1 & 0 \\
\hline 57 & 4 & 1 & 1 & 1 & 0 & 1 & 0 \\
\hline 58 & 10 & 2 & 3 & 2 & 2 & 1 & 0 \\
\hline 59 & 2 & 0 & 1 & 1 & 0 & 0 & 0 \\
\hline 60 & 6 & 1 & 2 & 1 & 1 & 1 & 0 \\
\hline 61 & 6 & 1 & 2 & 1 & 1 & 1 & 0 \\
\hline 62 & 6 & 1 & 2 & 1 & 1 & 1 & 0 \\
\hline
\end{tabular}

\begin{tabular}{|c|c|c|c|c|c|c|c|}
\hline 63 & 1 & 0 & 0 & 0 & 1 & 0 & 0 \\
\hline 64 & 3 & 0 & 1 & 0 & 1 & 1 & 0 \\
\hline 65 & 9 & 2 & 3 & 1 & 1 & 2 & 0 \\
\hline 66 & 5 & 2 & 1 & 1 & 0 & 1 & 0 \\
\hline 67 & 3 & 0 & 1 & 0 & 1 & 1 & 0 \\
\hline 68 & 7 & 1 & 3 & 2 & 0 & 1 & 0 \\
\hline 69 & 3 & 0 & 1 & 0 & 1 & 1 & 0 \\
\hline 70 & 5 & 2 & 1 & 1 & 0 & 1 & 0 \\
\hline 71 & 3 & 0 & 1 & 0 & 1 & 1 & 0 \\
\hline 72 & 5 & 2 & 1 & 1 & 0 & 1 & 0 \\
\hline 73 & 9 & 2 & 3 & 1 & 1 & 2 & 0 \\
\hline 74 & 1 & 0 & 0 & 0 & 1 & 0 & 0 \\
\hline 75 & 5 & 2 & 1 & 1 & 0 & 1 & 0 \\
\hline 76 & 3 & 0 & 1 & 0 & 1 & 1 & 0 \\
\hline 77 & 9 & 2 & 3 & 1 & 1 & 2 & 0 \\
\hline 78 & 2 & 0 & 1 & 1 & 0 & 0 & 0 \\
\hline 79 & 1 & 0 & 0 & 1 & 0 & 0 & 0 \\
\hline 80 & 10 & 3 & 3 & 1 & 2 & 1 & 0 \\
\hline 81 & 1 & 1 & 0 & 0 & 0 & 0 & 0 \\
\hline 82 & 10 & 2 & 3 & 1 & 2 & 2 & 0 \\
\hline 83 & 2 & 0 & 1 & 1 & 0 & 0 & 0 \\
\hline 84 & 6 & 1 & 2 & 1 & 1 & 1 & 0 \\
\hline 85 & 9 & 2 & 3 & 1 & 1 & 2 & 0 \\
\hline 86 & 8 & 2 & 3 & 1 & 0 & 2 & 0 \\
\hline 87 & 6 & 1 & 2 & 1 & 1 & 1 & 0 \\
\hline 88 & 9 & 2 & 3 & 1 & 1 & 2 & 0 \\
\hline 89 & 3 & 0 & 1 & 0 & 1 & 1 & 0 \\
\hline 90 & 1 & 0 & 0 & 0 & 1 & 0 & 0 \\
\hline 91 & 2 & 0 & 1 & 1 & 0 & 0 & 0 \\
\hline 92 & 2 & 0 & 1 & 1 & 0 & 0 & 0 \\
\hline 93 & 3 & 0 & 1 & 0 & 1 & 1 & 0 \\
\hline 94 & 11 & 2 & 4 & 1 & 1 & 2 & 1 \\
\hline 95 & 1 & 0 & 1 & 0 & 0 & 0 & 0 \\
\hline 96 & 11 & 2 & 4 & 1 & 1 & 2 & 1 \\
\hline 97 & 3 & 0 & 1 & 0 & 1 & 1 & 0 \\
\hline 98 & 9 & 2 & 3 & 1 & 1 & 2 & 0 \\
\hline 99 & 11 & 2 & 4 & 1 & 1 & 2 & 1 \\
\hline 100 & 4 & 1 & 1 & 1 & 0 & 1 & 0 \\
\hline
\end{tabular}

Tabla 4 - Cuantitativo total de defectos conforme clasificación.

\begin{tabular}{|c|c|c|c|}
\hline Defeitos & N.o defeitos & \% Individual & \% Acumulada \\
\hline Tipo 2 & 191 & $34 \%$ & $34 \%$ \\
\hline Tipo 5 & 110 & $20 \%$ & $53 \%$ \\
\hline Tipo 1 & 103 & $18 \%$ & $72 \%$ \\
\hline Tipo 3 & 90 & $16 \%$ & $88 \%$ \\
\hline Tipo 4 & 59 & $10 \%$ & $98 \%$ \\
\hline Outros & 11 & $2 \%$ & $100 \%$ \\
\hline Total & 564 & \multicolumn{2}{|l}{} \\
\hline
\end{tabular}

Fuente: Los Autores, (2019).

A partir de las informaciones, se puede trazar el gráfico de Pareto, que nos proporciona una mejor visualización de los 3 principales problemas. El gráfico se muestra en la Figura 6
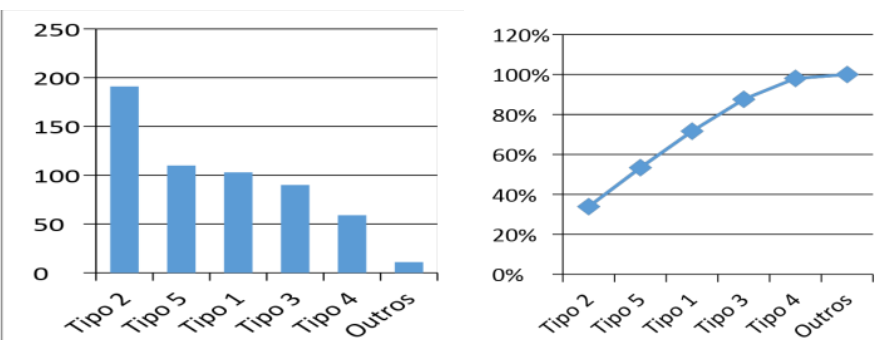

Figura 6 - Gráfico de Pareto.

Fuente: Los Autores, (2019). 
Observando el gráfico, verificando que los defectos Tipo 2, 5 y 1 son los que más contribuyen a que las muestras queden fuera de la especificación. Para actuar en el proceso, fue necesario el levantamiento del diagrama de flujo, para identificar las actividades relacionadas con los principales defectos presentados.

Los problemas Tipo 1 y 2 estaban relacionados con el operador de celular, el cual fue resuelto sustituyendo por otro operador con mayor calidad en el servicio. En el caso del defecto tipo 5, que es antena desconectada, se ha reforzado la conexión con un tipo de adhesivo durante el período de Life Test.

Para verificar si la solución fue eficiente, se recolectar nuevas muestras, de sólo 10 semanas, cuyos resultados están de acuerdo con lo presentado en la Tabla 5.

Tabla 5 - Levantamiento de defectos después de aplicada la acción de mejora.

\begin{tabular}{|c|c|c|}
\hline Semana & Defectos & Grupo de Muestras \\
\hline 1 & 5 & 200 \\
\hline 2 & 11 & 200 \\
\hline 3 & 3 & 200 \\
\hline 4 & 11 & 200 \\
\hline 5 & 5 & 200 \\
\hline 6 & 5 & 200 \\
\hline 7 & 7 & 200 \\
\hline 8 & 3 & 200 \\
\hline 9 & 3 & 200 \\
\hline 10 & 7 & 200 \\
\hline \multicolumn{2}{|c|}{ Fuente. Los Autores, (2019). }
\end{tabular}

Aplicando nuevamente la carta $\mathrm{P}$ y $\mathrm{PN}$, se verifica que el proceso está bajo control estadístico, como se muestra en el gráfico de la Figura 8.

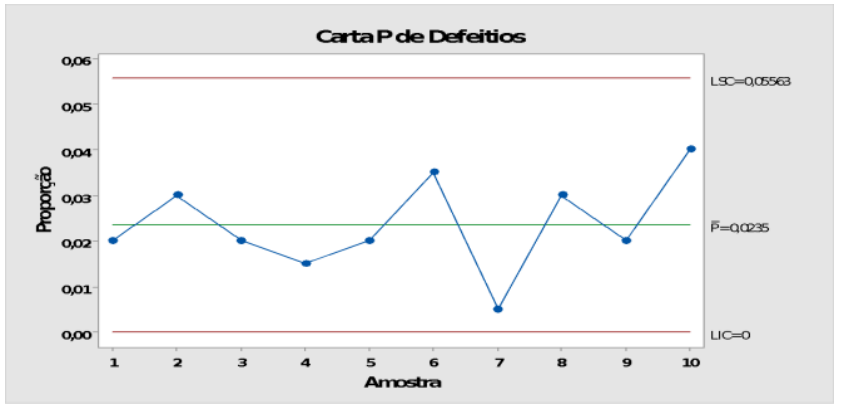

Figura 7 - Gráfico de control P.

Fuente: Los Autores, (2019).

Gráfico de control P en los datos recolectados después de la acción en el proceso.

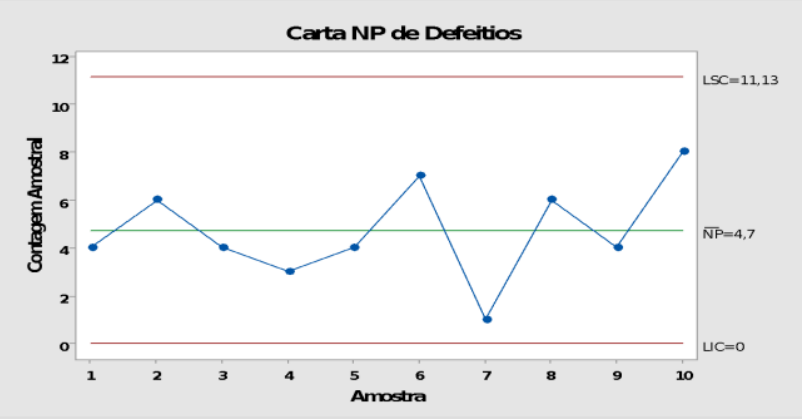

Figura 8 - Gráfico de control PN. Fuente: Los Autores, (2019)
Gráfico de control PN en los datos recolectados después de la acción en el proceso. Aplicando nuevamente la carta P y PN, verifica-se el proceso es capaz, se utilizó el límite superior en el gráfico de control NP según lo especificado por el cliente.

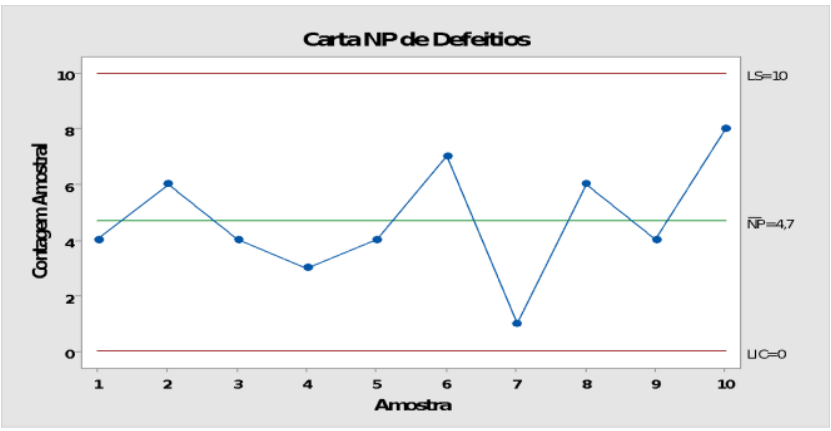

Figura 9 - Gráfico de control NP - Proceso capaz. Fuente: Los Autores, (2019).

El resultado se muestra en la Figura 9, ilustrando que el proceso es capaz.

\section{CONCLUSIONES}

Las causas de no conformidades encontradas en el proceso, fueron solucionadas a medida que fueron identificadas, en cierto modo se atendió a las necesidades del cliente ya los objetivos de la empresa. Los ajustes de calidad con la implantación del CEP en la empresa, hacen que el producto hoy esté atendiendo a las especificaciones. Con las medidas se detectó que ya no hay más grandes números de muestras siendo devueltas y la empresa disminuyó considerablemente las no conformidades. De esta forma, la aplicación del CEP en la empresa es extremadamente importante, y se hace necesario. Habiendo sido adoptado a partir de la investigación en todo el proceso productivo.

\section{REFERENCIAS}

[1] Campos, Emerson de Souza; Figueiredo, Luiz; Meneses, Jose; Dantas, Maria José Pereira; CUNHA, Victor Honorato. Controle estatístico de processo em uma indústria de transformadores elétricos: um estudo de caso. XII Congresso Nacional de Excelência em Gestão \& III Inovarse-Responsabilidade Social Aplicada. São Paulo, 2016.

[2] Dias, Mallvina; Amorim, Lidiane de Souza; Bueno, Alex Alves; Pereira, Narallyne Kelly de Castro; Alves, Alisson Lucas. Utilização de CEP para análise da prestação de serviços em uma empresa de transporte público. XXXII Encontro Nacional de Engenharia de Produção Desenvolvimento Sustentável e Responsabilidade Social: As Contribuições da Engenharia de Produção. Bento Gonçalves, RS, Brasil, 2012.

[3] Fonseca, Patrícia Corrêa. Modelo para controle estatístico de processos de desenvolvimento de software (CEP-S), 2010, p.35. Dissertação (Mestrado em Ciência da Computação), Coordenação Pós-Graduação em Ciência da Computação do Instituto de Ciências Exatas da Universidade Federal de Minas Gerais. Belo Horizonte.

[4] Goncalves, Patrícia; Maria, Diene; Goncalves, Nayane; Melo, Luciana Vieira de; SOARES, Júlio Cesar Valandro. Aplicação do 
Cep na Prestação de Serviços de Internet. XXXV Encontro Nacional de Engenharia de Produção Perspectivas Globais para a Engenharia de Produção Fortaleza, CE, Brasil, 2015.

[5] Lopes, Oswaldo. Tecnologia Mecânica. Edgard Blucher. SP 1983. 180p.

[6] Morais, Tércius Cassius Melo de; SÁ, Sergio Roque; Fonseca, Márcio Botelho da. Aplicações do controle estatístico de processo para o controle de tensão elétrica em subestações: estudo de caso de uma empresa distribuidora de energia elétrica. XXX encontro nacional de engenharia de produção: Maturidade e desafios da Engenharia de Produção: competitividade das empresas, condições de trabalho, meio ambiente. Enegep: São Carlos, SP, Brasil, 2010.

[7] Noskievicová, Darja. Effective implementation of SPC. VŠBTU Ostrava. Czech Republic, 2010.

[8] Oliveira. Camila Cardoso de. Granato, Daniel. Caruso, Miriam Solange Fernandes. Sakuma, Alice Momoyo. Manual para elaboração de cartas de controle para monitoramento de processos de medição quantitativos em laboratórios de ensaio. $1^{a}$ edição São Paulo - SES/SP 2013.

[9] Stamatis D. H. Six Sigma and Beyond: Statistical Process Control, Volume IV. Lucie Press. 2003.

[10] Silva, Rouverson p., Souza, Fábio G., Cortez, Jorge W.; Furlani, Carlos E. A., Vigna Gustavo P. Variabilidade espacial e controle estatístico do processo de perdas na colheita mecanizada do algodoeiro, Pg 5, Eng. Agric., Jaboticabal, v.27, n.3, p.742-752, set./dez.2007. 